\title{
Specificity and heregulin regulation of Ebp I (ErbB3 binding protein I) mediated repression of androgen receptor signalling
}

\author{
Y Zhang' and AW Hamburger ${ }^{*, 1,2}$ \\ 'Greenebaum Cancer Center, University of Maryland School of Medicine, Baltimore, MD, USA; ${ }^{2}$ Department of Pathology, University of Maryland School \\ of Medicine, Baltimore, MD, USA
}

\begin{abstract}
Although ErbB receptors have been implicated in the progression of prostate cancer, little is known about proteins that may mediate their interactions with the androgen receptor (AR). Ebpl, a protein cloned via its association with the ErbB3 receptor, binds the AR and inhibits androgen-regulated transactivation of wild-type AR in COS cells. As the complement of coregulators in different cells are important for AR activity, we determined the effect of Ebp I on AR function in prostate cancer cell lines. In addition, we examined the regulation of Ebp I function by the ErbB3/4 ligand heregulin (HRG). In this study, we demonstrate, using several natural AR-regulated promoters, that Ebpl repressed transcriptional activation of wild-type AR in prostate cancer cell lines. Downregulation of Ebp I expression in LNCaP cells using siRNA resulted in activation of AR in the absence of androgen. Ebpl associated with ErbB3 in LNCaP cells in the absence of HRG, but HRG induced the dissociation of Ebp I from ErbB3. In contrast, HRG treatment enhanced both the association of Ebp I with AR and also the ability of Ebpl to repress AR transactivation. These studies suggest that Ebp I is an AR corepressor whose biological activity can be regulated by the ErbB3 ligand, HRG.

British Journal of Cancer (2005) 92, |40- |46. doi: I0.1038/sj.bjc.6602257 www.bjcancer.com

Published online 7 December 2004

(c) 2005 Cancer Research UK
\end{abstract}

Keywords: prostate cancer; androgen receptor; ErbB receptors; Ebp I

Prostate cancer is the second most prevalent cancer among men in the United States and ranks second to lung cancer in terms of annual mortality (Weir et al, 2003). Prostate cancer begins as an androgen-dependent tumour that undergoes clinical regression in response to pharmacological and surgical strategies that reduce testosterone concentration. Despite this treatment, the cancer eventually regrows as an androgen- or hormone-independent tumour (Feldman and Feldman, 2001). Microarray analysis of both androgen dependent and independent tumour xenografts (Amler et al, 2000; Mousses et al, 2001) and human prostate cancer samples (LaTulippe et al, 2002) during disease progression has identified several candidate targets, including the AR itself, for prostate cancer therapy and diagnosis associated with the androgen independent phenotype. Thus, aberrant changes in AR signalling are likely to play a role in the progression to androgen independence (Grossmann et al, 2001). Chen et al (2004) showed, using microarray-based profiling of isogenic prostate cancer xenografts, that increases in AR mRNA were the only changes consistently associated with development of resistance to antiandrogen therapy, providing a strong rationale for targeting the downregulation of androgen receptor (AR) activity in the treatment of advanced prostate cancer.

The potential role of the epidermal growth factor (ErbB) family of receptors and their ligands in regulating AR activity during prostate cancer progression is currently a focus of intense

\footnotetext{
* Correspondence: Dr A Hamburger, University of Maryland Cancer Center, Greenebaum Cancer Center, 655 W. Baltimore St, Rm 9-0046 BRB, Baltimore, MD 2120I, USA; E-mail: ahamburg@umaryland.edu Received 27 May 2004; revised 5 October 2004; accepted 18 October 2004; published online 7 December 2004
}

investigation. This receptor family includes four members: EGFR (ErbB1), ErbB2 (Neu, HER2), ErbB3 (Her3) and ErbB4 (Her4). All EGFR family members contain an extracellular ligand binding domain, a transmembrane region important in regulating receptor activity, and a cytoplasmic tyrosine kinase domain. ErbB3 lacks tyrosine kinase activity due to amino-acid substitutions in the conserved kinase domain (Kirschbaum and Yarden, 2000). ErbB receptors have been implicated in the pathogenesis and progression of many types of human cancers and therapies directed against these receptors are in clinical use (Yarden, 2001). An extensive body of work demonstrating cross talk between ErbB receptors and their ligands and the $\mathrm{AR}$ in prostate cancer has evolved (El Sheikh et al, 2003). For example, the EGF receptor is overexpressed in both benign prostatic hypertrophy (BPH) and prostate cancer (De Miguel et al, 1999). EGFR overexpression is observed in patients during a conversion to androgen independent growth (Olapade-Olaopa et al, 2000; Hernes et al, 2004). The role of ErbB2 in rendering cells androgen independent or more sensitive to extremely low levels of androgen has been demonstrated both in vitro and in animal models (Craft et al, 1999; Yeh et al, 1999). ErbB2 is also required for IL-6 activation of AR signalling (Qiu et al, 1998). However, studies of the role of ErbB2 in clinical prostate cancer remain inconclusive (Grossmann et al, 2001), and initial clinical trials indicate that the anti-ErbB2 antibody Herceptin (trastuzumab) does not show significant clinical activity as a single agent (Ziada et al, 2004). ErbB3 has not been as extensively studied, but analysis of clinical prostate cancer specimens indicates that overexpression of ErbB3 has been linked to a less favourable prognosis (Leung et al, 1997).

EGF-like ligands have been shown in vitro and in vivo to stimulate growth of prostate cancer cells. For example, in vitro AR is activated in a ligand independent manner by EGF (Culig et al, 
1994). Torring et al (2003) recently showed that LNCaP cells constitutively express EGF ligands and that ErbB1 activity is necessary for androgen-induced proliferation. Endogenous stromal derived factors such as Heparin binding-EGF attenuate the response of $\mathrm{AR}$ to its ligands, resulting in androgen independent growth of LNCaP cells (Adam et al, 2002). By contrast, the ErbB3/4 ligand HRG is highly expressed in basal, luminal and stromal cells of the normal prostate, but not in prostate tumours (Lyne et al, 1997). Further, HRG protein is not detected in the prostate cancer cell lines LNCaP, DU-145 or PC-3 (Grasso et al, 1997a; Lyne et al, 1997). HRG mRNA could be detected in these cell lines only by RT - PCR in one study (Lyne et al, 1997), but was not detected in another (Grasso et al, 1997a). HRG inhibits growth and induces differentiation of AR positive, ErbB1-3 positive LNCaP cells, but has little effect on proliferation of ErbB1-3 positive, AR negative DU 145 and PC-3 cells (Grasso et al, 1997b; Lyne et al, 1997). In addition, HRG induces the expression of the tumour suppressor p53 and the CDK inhibitor p21 in LNCaP cells (Bacus et al, 1996). Most recently, Tal-Or et al (2003) have demonstrated that HRG activates ErbB2/3 heterodimers and induces apoptosis of $\mathrm{LNCaP}$ cells. These combined findings suggest that HRG signals may contribute to growth restriction or differentiation of prostate epithelia.

Our laboratory has recently demonstrated that a protein Ebp1, isolated by its binding to HRG's cognate receptor ErbB3 (Yoo et al, 2000 ), binds AR in vitro and in vivo (Zhang et al, 2002). Ebp1 is expressed in both normal prostate epithelial cells and in the prostate cancer cell lines LNCaP, DU-145 and PC-3 (Xia et al, 2001b). Ectopic expression of ebp1 inhibits ligand-mediated transcriptional activation of both artificial and natural AR regulated promoters in COS cells transfected with wild-type AR and in LNCaP cells that express a mutant AR. The transcription of the endogenous PSA gene is also decreased in LNCaP cells stably transfected with Ebp1 (Zhang et al, 2002). However, the effect of Ebp1 on transactivation of wild-type AR in prostate cancer cells was not established. The purpose of the present study was to extend our finding that Ebp1 represses AR transactivation and to determine if the ErbB3 ligand, HRG, affects Ebp1's interactions with AR

\section{MATERIALS AND METHODS}

\section{Cell culture}

All cell lines except PC-3 AR were obtained from the American Type Culture Collection (Manassas, VA, USA). PC-3 AR cells (Long et al, 2000) were a gift of Dr Angela Brodie. Cells were maintained at $37^{\circ} \mathrm{C}$ in a humidified atmosphere of $5 \% \mathrm{CO}_{2}$ in air. Cell lines were routinely cultured in RPMI 1640 media supplemented with $10 \%$ foetal bovine serum (FBS) (Sigma, St Louis, MO, USA).

\section{Plasmids}

The PSA reporter luciferase construct was a gift from Dr Martin Gleave and contains $-630 /+12$ of the $5^{\prime}$ PSA flanking region. The Probasin $(-285 /+32)$ luciferase reporter and the pSG5-hAR expression construct were gifts of Dr O Janne. The MMTVluciferase plasmid was obtained from Dr Joseph Fondell (Wang and Fondell, 2001). The ebp1 expression construct has been previous described (Xia et al, 2001a).

\section{Immunoprecipitation, GST-pulldowns and Western blot analysis}

To measure ErbB3 - Ebp1 interactions, LNCaP cells were incubated overnight in serum-free RPMI-1640 media. Where indicated, cells were treated with $20 \mathrm{ng} \mathrm{ml}^{-1}$ of HRG $\beta 1$ (R\&D Systems, Mpls, MN,
USA) for the indicated times. Cell lysates were prepared and immunoprecipitated as described previously (Fernandes et al, 1999). Briefly, cells were lysed with buffer containing $50 \mathrm{~mm}$ HEPES ( $\mathrm{pH} 7.5$ ), $1 \mathrm{~mm}$ EDTA, $150 \mathrm{~mm} \mathrm{NaCl}, 1 \%$ Triton X-100 and Complete ${ }^{\mathrm{TM}}$ protease inhibitor. Protein concentrations were measured using a detergent compatible kit (BioRad, Hercules, CA, USA). Cell lysates were precleared with Protein A/Protein G agarose and immunoprecipitated for $4 \mathrm{~h}$ at $4{ }^{\circ} \mathrm{C}$ with $2 \mu \mathrm{g}$ of a monoclonal antibody directed against ErbB3 (Santa Cruz Biotechnology, Santa Cruz, CA, USA) and $20 \mu \mathrm{l}$ packed Protein A/G agarose beads. The immunoprecipitates were washed and resuspended in Laemmli sample buffer. Proteins were resolved by SDSPAGE. After electrophoresis, the proteins were transferred onto Immobilin-P membranes, and immunoblotted as described (Xia et al, 2001b) using a monoclonal antibody to ErbB3 (Santa Cruz) or a rabbit polyclonal antibody that detects both phosphorylated and unphosphorylated forms of Ebp1 (Xia et al, 2001b) (Upstate, Lake Placid, NY, USA). To measure the association of endogenous $\mathrm{AR}$ and endogenous Ebp1, LNCaP cells, growing in complete media, were switched to phenol-red free RPMI 1640 containing $1 \%$ charcoal stripped calf serum (CSS) (Sigma) and $10^{-8}{ }_{\mathrm{M}} \mathrm{R} 1881$ (NEN, Boston, MA, USA) for $24 \mathrm{~h}$. Cells were then stimulated with or without HRG $\beta 1$ (R\&D Systems, Mpls, MN, USA) for $1 \mathrm{~h}$. Cell lysates were immunoprecipitated as described above using the polyclonal antibody to Ebp1. Western blot analysis was performed using a monoclonal antibody to AR (Santa Cruz) or the Ebp1 antibody.

\section{Luciferase reporter assays}

Cells $\left(5 \times 10^{4}\right)$ were plated in 12 -well plates in complete media. When cells reached 50-60\% confluence, they were transfected using the Fugene-6 Reagent (Roche, Indianapolis, IN, USA) according to the manufacturer's instructions. Cells were transfected with $0.5 \mu \mathrm{g}$ of the indicated reporter plasmids, $0.5 \mu \mathrm{g}$ of pSG5-hAR (where specified), and $0.5 \mu \mathrm{g}$ of pcDNA3 or wild-type ebp1 expression plasmids and $5 \mathrm{ng}$ of the TK-Renilla plasmid (Promega, Madison, WI, USA) as an internal control. Complete medium was replaced $24 \mathrm{~h}$ after transfection with phenol red free RPMI 1640 with CSS with or without R1881 $\left(10^{-8}\right.$ M) (Sadar and Gleave, 2000). Luciferase activity was determined using the Promega Dual luciferase assay kit as described by the manufacturer. The levels of luciferase activity were normalised using the renilla luciferase as an internal control. The ratio of luciferase activity to the renilla control derived from cells that were transfected with vector alone and not treated was given a Relative Luciferase Activity value of 1 . All values presented in the individual figures were derived by comparison to this ratio observed in control cells. Transfection efficiency was approximately $30 \%$ as judged by parallel experiments using the EGFP-N1 plasmid (Clontech, Palo Alto, CA, USA). All transfection experiments were carried out in triplicate wells.

\section{Gene silencing with small interfering RNAs}

The siRNA oligonucleotides were purchased from Dharmacon Research Inc (Lafayette, CO, USA). COS-7 cells were cultured in 12 -well plates until $60 \%$ confluent. Cells in $1 \mathrm{ml}$ of antibiotic-free culture media were transfected with $60 \mathrm{~nm}$ final concentration of annealed oligonucledotides using Lipfectamine 2000 according to the manufacturer's instructions. The Ebp1 siRNA sequences corresponded to the coding regions beginning at nucleotides 476 and 995 (Genbank accession number U87954). The targets sequences were AAGCGACCAGGAUUAUAUUCU and AAGUGAGGUGGAAAGGCGUUU respectively. These sequences do not match any other human genomic sequences as determined by BLAST analysis using the NCBI Website. Scrambled oligonucleotides of these sequences were used as negative controls. The 
next day, cells were transfected with an expression construct for wild-type AR and the MMTV-luciferase and TK plasmids using Fugene-6.

\section{Statistical analysis}

Results were analysed using a two-tailed Studentst-test. Significance was established at $P \leqslant 0.05$.

\section{RESULTS}

\section{Ebp1 inhibits transactivation of wild-type AR in prostate} adenocarcinoma cell lines

We previously demonstrated that Ebp1 inhibited transactivation of the artificial $\mathrm{ARE}_{2} \mathrm{DS}$ promoter in COS cells transfected with wildtype $\mathrm{AR}$, and the PSA promoter in LNCaP cells that harbor a mutant AR (Zhang et al, 2002). We were interested in determining if Ebp1 represses wild-type AR in prostate cancer cell lines. We therefore tested the ability of Ebpl to repress AR-mediated transcription of the MMTV-luciferase reporter plasmid in androgen-independent DU145 cells transiently transfected with a wildtype AR and PC-3 AR cells stably transfected with wild-type AR (Long et al, 2000). Cells were transfected with the MMTV luciferase reporter construct, ebp1 or the pcDNA vector control, and in the case of DU145 cells, the expression construct for AR. After $24 \mathrm{~h}$, the cells were stimulated with either R1881 or vehicle for $16 \mathrm{~h}$. Cells were harvested and monitored for dual luciferase activity. Transfection of $e b p 1$ at $0.5 \mu \mathrm{g}$ per individual well routinely results in a two- to three-fold increase in Ebp1 expression levels as determined by Western blot analysis (data not shown). As expected, R1881 stimulated luciferase activity four- to five-fold in DU145 and PC-3 AR cells (Figure 1). Ectopic expression of ebp1 reduced $A R$ transactivation to basal levels in both cell types.

Corepressors may have dissimilar effects on the activity of natural AR regulated promoters due to differential binding of the $\mathrm{AR}$ to androgen response elements within those promoters (Claessens et al, 2001). We had previously demonstrated that Ebp1 represses both exogenous and endogenous PSA promoter activity in LNCaP cells (Zhang et al, 2002). We therefore tested the ability of Ebp1 to repress the MMTV-luc and probasin native androgen responsive promoters. LNCaP cells were stimulated with $\mathrm{R} 1881$ and the induction of luciferase activity in the presence and absence of exogenous ebp1 was measured. Probasin and MMTV promoters were strongly activated by R1881. AR activation of both promoters was reduced significantly $(P \leqslant 0.05)$ with the ectopic expression of ebp1 (Figure 2). However, a small but significant increase in AR activity after R1881 treatment was noted even in the presence of $e b p 1$.

We next wished to determine if endogenous Ebp1 was important in AR signalling. COS-7 cells were first transfected with siRNA targeted to two regions in the Ebp1 cDNA as described in the Materials and Methods. Cells were then transfected with the AR expression plasmid, and the MMTV-luciferase reporter construct the next day. Cells were stimulated with R1881 on day 3 and lysates collected on day 4 . The results of Western blotting experiments showed that transfection of siRNA directed against Ebp1 reduced proteins levels about $80 \%$ at Day 4 (Figure $3 \mathrm{~A}$ ). This decrease was not observed in cells transfected with scrambled oligos. Decreased expression of Ebp1 resulted in a significant $(P \leqslant 0.05) 3.5$-fold increase in the luciferase activity of the MMTV promoter in the absence of androgen. No such stimulation was observed in cells lacking AR. R1881 stimulation of the reporter plasmid was decreased by inhibition of Ebp1 expression, but this change was not significant at the $P \leqslant 0.05$ level (Figure 3B). These results suggest that Ebp1 may be important in repression of AR in the absence of androgen.
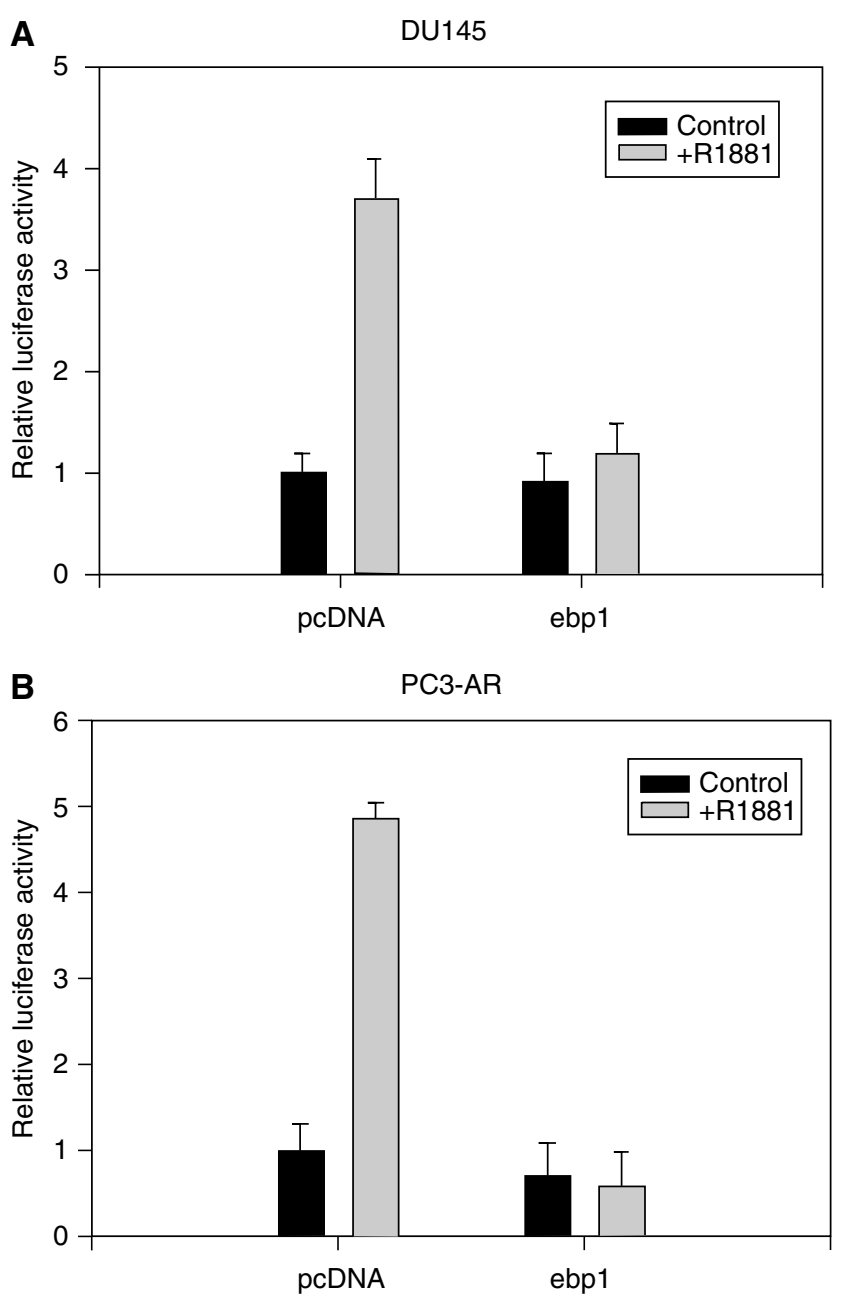

Figure I Ebpl inhibits the transcriptional activity of the MMTV promoter in prostate cancer cell lines expressing wild-type AR DUI45 (A) or PC3-AR cells (B) were transfected with an MMTV-luciferase reporter plasmid and pcDNA or pcDNA-ebp / expression plasmids where indicated. DUI45 cells were also transfected with a wild-type AR expression plasmid. At $24 \mathrm{~h}$ after transfection, cells were switched to phenol-red free RPMI 1640 media with I\% CSS containing RI 88I (I0-8 M) or vehicle control. After $16 \mathrm{~h}$, luciferase activity was measured. Each point represents mean \pm s.e. of triplicate wells. Representative of three experiments.

\section{HRG regulates the binding of Ebp1 to ErbB3 and AR}

We next determined if ErbB3 could bind Ebp1 in human prostate cell lines as it does in breast carcinoma cells (Yoo et al, 2000) and if HRG could affect this binding. Lysates of serum starved LNCaP cells were incubated with either a mouse monoclonal antibody to ErbB3 or control IgG. Proteins were resolved by SDS-PAGE and immunoblotted with antibody to Ebp1. Ebp1 was found in ErbB3, but not control, immunoprecipitates (Figure 4A). Next, we determined if the binding of Ebp1 to ErbB3 could be regulated by HRG. LNCaP cells were serum starved and treated with HRG $\left(20 \mathrm{ng} \mathrm{ml}^{-1}\right)$ for $0,15,60$ and $120 \mathrm{~min}$ and $24 \mathrm{~h}$. Cell lysates were immunoprecipitated with antibody to ErbB3 as described. Ebp1 was found in ErbB3 immunoprecipitates of untreated cells (Figure 4B). There was a decrease in the level of Ebp1 associated with ErbB3 starting at $15 \mathrm{~min}$. No Ebp1 was found in the Erb3 immunoprecipitates $60 \mathrm{~min}$ after treatment. Binding was increased $2 \mathrm{~h}$ after treatment and by $24 \mathrm{~h}$ after HRG treatment, Ebp1 binding to ErbB3 was restored. 
A

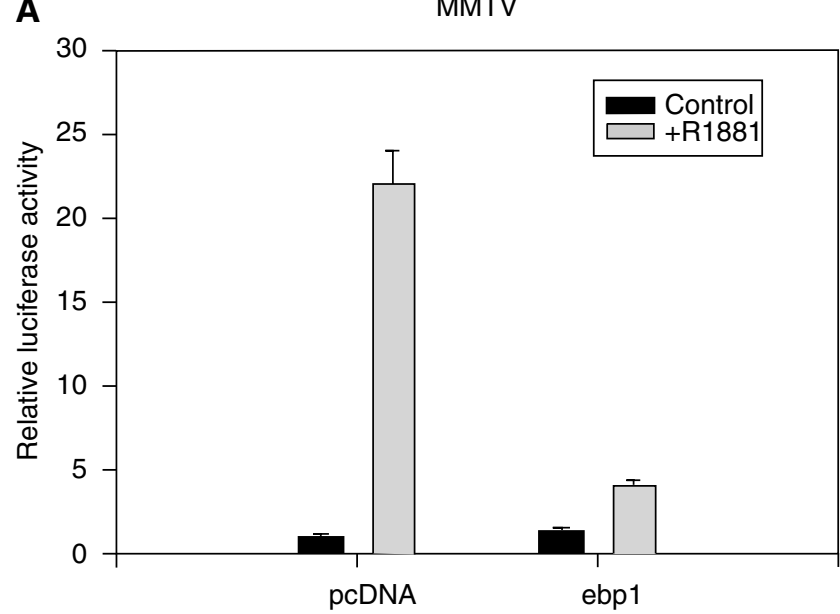

B

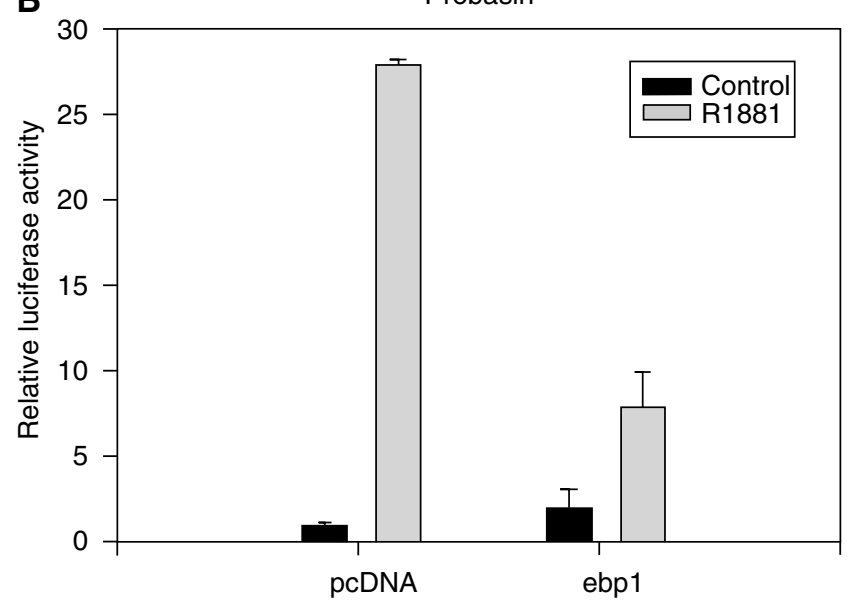

Figure 2 Ebpl inhibits transcriptional activity of native $A R$ target promoters in LNCaP cells. LNCaP cells were transfected with the MMTV (A) or probasin reporter constructs $(\mathbf{B})$ and $0.5 \mu \mathrm{g}$ of pcDNA3 or the wild-type ebp / expression plasmid. At $24 \mathrm{~h}$ after transfection, cells were treated with RI88I as described in Figure I for an additional $16 \mathrm{~h}$ and relative luciferase activity was measured. Each point represents mean \pm s.e. of triplicate wells. Representative of two experiments.

As HRG treatment resulted in the release of Ebp1 from ErbB3, we next determined whether HRG could regulate the association of endogenous Ebp1 with endogenous AR. LNCaP cells, growing in complete media, were switched into phenol-red free RPMI 1640 media with $1 \%$ CSS and R1881 $\left(10^{-8} \mathrm{M}\right)$ overnight. Cells were then treated with $20 \mathrm{ng} \mathrm{ml}^{-1}$ of HRG $\beta 1$ for $1 \mathrm{~h}$, a time when we could not detect Ebp1 in ErbB3 immunoprecipitates. Cell lysates were immunoprecipitated with the Ebp1 antibody. Western blot analysis of the immunoprecipitates indicated that HRG treatment enhanced the interaction of Ebp1 with AR (Figure 5A). Examination of cell lysates revealed that HRG treatment did not increase the level of AR protein at this $1 \mathrm{~h}$ time point (Figure $5 \mathrm{~B}$ ).

HRG enhances Ebp1 transcriptional repression. We reasoned that if HRG could change the association of Ebpl with AR, it could affect Ebp1 induced repression of AR transactivation. LNCaP cells were transiently transfected with the MMTV luciferase reporter plasmid and limiting amounts of an ebp1 expression construct. $E b p 1$ at low concentrations $(0.1 \mu \mathrm{g})$ reduced AR luciferase activity $55 \%$. Maximal inhibition of $90 \%$ was observed at $0.5 \mu \mathrm{g}$ of the $e b p 1$ plasmid. This was more than the $80 \%$ inhibition previously observed (Figure 1) and probably due to changes in transfection
A
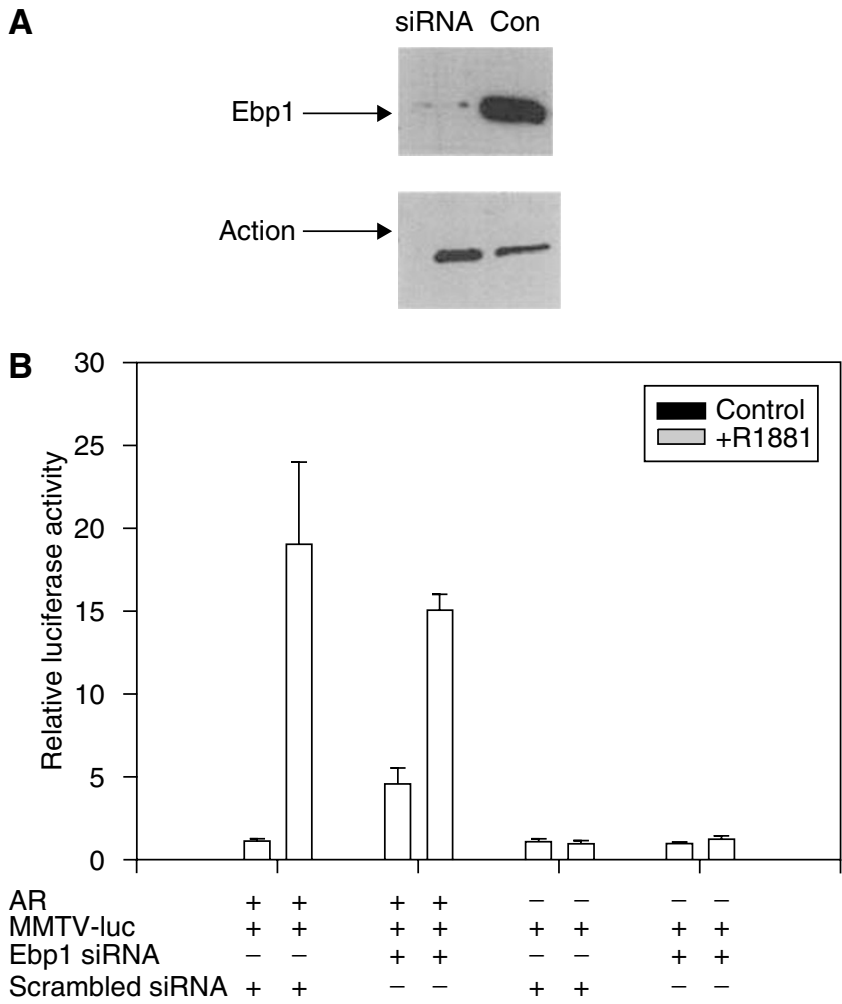

Figure 3 The effect of Ebpl specific small interfering RNA on transactivation of AR in COS-7 cells. COS-7 cells were transfected with scrambled siRNAs (Con) or siRNAs (siRNA) directed towards ebp I cDNA sequences 476 and 995 using Lipofectamine 2000. The next day the cells were transfected with the wild-type AR expression plasmid, MMTVluc and RL-TK. After $24 \mathrm{~h}$, the cells were switched to phenol-red free RPMI 1640 media with 5\% CSS with or without RI88I. Cell lysates were harvested for Western blot analysis and luciferase activity $16 \mathrm{~h}$ later. (A) The expression of Ebp I was analysed by Western blotting. Cell lysates were resolved by SDS-PAGE, proteins transferred onto PVDF membranes and filters blotted with Ebpl or actin antibodies as indicated. (B) Effect of Ebpl siRNA on AR promoter activity. Aliquots of the cells harvested in $(\mathbf{A})$ were assayed for luciferase activity. Each point represents mean \pm s.e. of triplicate wells. Representative of three experiments.

efficiencies as different batches of cells and plasmids were used in these different experiments. Concentrations of HRG $\left(20 \mathrm{ng} \mathrm{ml}^{-1}\right)$, previously demonstrated to increase association of Ebp1 and AR, significantly $(P \leqslant 0.05)$ enhanced Ebp1-mediated repression at low $(0.1$ and $0.2 \mu \mathrm{g})$ amounts of the Ebp1 plasmid (Figure 6).

\section{DISCUSSION}

We have previously established that Ebp1, a protein cloned in our laboratory via its interactions with the ErbB3 receptor, inhibits $\mathrm{AR}$-mediated transcription and growth of the AR positive $\mathrm{LNCaP}$ cell line (Zhang et al, 2002). In this report, we confirm and extend our findings by demonstrating that Ebp1 is capable of inhibiting receptor transactivation independent of cell type or AR target promoter. In addition, we demonstrate that HRG, the ErbB3 ligand, stimulated the association of Ebpl with $\mathrm{AR}$ and increased Ebp1 mediated repression of AR activity, providing further evidence for a link between ErbB ligands and AR function.

This study first demonstrated that Ebp1 inhibition of $A R$ transactivation was neither promoter nor cell type specific. We had previously demonstrated that Ebpl inhibits AR transactivation of 
A

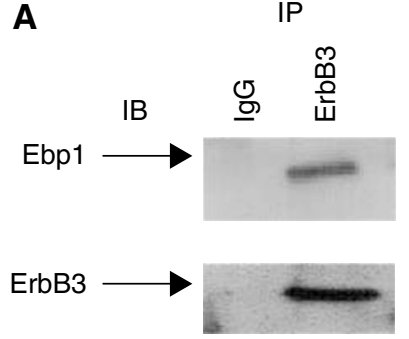

B
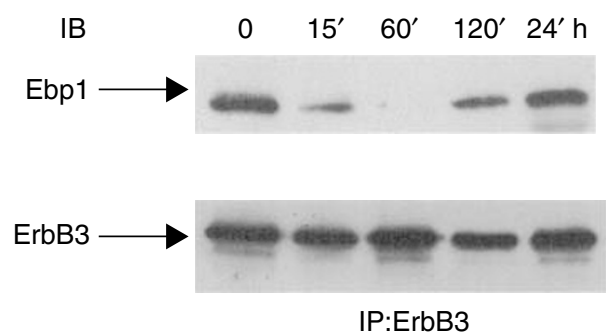

Figure 4 In vivo interaction of Ebp I with $\operatorname{ErbB3}(\mathbf{A})$ Ebp I associates with ErbB3 in LNCaP cells. LNCaP cell lysates were immunoprecipitated with mouse isotype control lgG (lane I) or an anti-ErbB-3 monoclonal antibody (lane 2) and analysed by sequential immunoblotting (IB) with either Ebp I (top panel) or ErbB3 (bottom panel) antibodies. (B) HRG-induced dissociation of Ebp I. LNCaP cells were left untreated $(0)$ or stimulated with HRG $\left(20 \mathrm{ng} \mathrm{ml}^{-1}\right)$ for the times indicated. ErbB-3 associated Ebpl was analysed by ErbB-3 immunoprecipitation (IP) and sequential immunoblotting (IB) with antibodies against EbpI (top) and ErbB-3 (bottom).

the artificial $\mathrm{ARE}_{2}$ luciferase reporter in COS cells and the PSA luciferase reporter in LNCaP cells (Zhang et al, 2002). However, recent studies have demonstrated that $\mathrm{AR}$ mediated gene transcription is influenced by the cell type examined (Kotaja et al, 2000; Claessens et al, 2001; Holter et al, 2002). For example, Holter et al (2002) have shown that DAX1 inhibition of PSA and the $\mathrm{ARE}_{2}$ reporter was more potent in COS-7 than HeLa cells. This variability has been attributed to the complement of transcription factors and coregulators in different cell types. Therefore, we examined AR transactivation of MMTV-luc in two androgenindependent prostate cancer cell lines, PC3 and DU145, that had been transfected with wild-type AR. Ebp1 inhibited AR-regulated transcription in these androgen-independent cells. Similarly, Cyclin D1 inhibits AR transactivation across a wide variety of both prostate and nonprostate derived cell lines (Petre-Draviam et al, 2003). In addition, a number of AR coregulators demonstrate promoter specificity. For example, ARIP3 enhances transcription from minimal AREs, yet represses the probasin promoter (Kotaja et al, 2000). Activation of both the PSA and probasin promoters requires interactions of the $\mathrm{N}$ and $\mathrm{C}$ terminal domains of $\mathrm{AR}$, but this association is not required for activation of MMTV (He et al, 2000). Thus, it was important to examine the effects of Ebp1 on different native promoters. Here we show that Ebp1 also inhibited the probasin and MMTV reporters in LNCaP cells as well as PSA as previously demonstrated (Zhang et al, 2002). Our previous studies had also shown that inhibition of AR transactivation is specific, as Ebp1 did not affect the estrogen induced responsiveness of an ERE luciferase reporter or the thyroid hormone mediated activity of a TRE luciferase reporter plasmid (Zhang et al, 2002). It is of interest to note that while ebp1 overexpression completely inhibited AR activity in DU145 and PC-3 AR transfected cells, ebp1 was unable to completely suppress the response to R1881 in LNCaP cells. This discrepancy may have been due to different expression levels of AR in the DU145 and PC-3 cells lines as compared to LNCaP cells, the

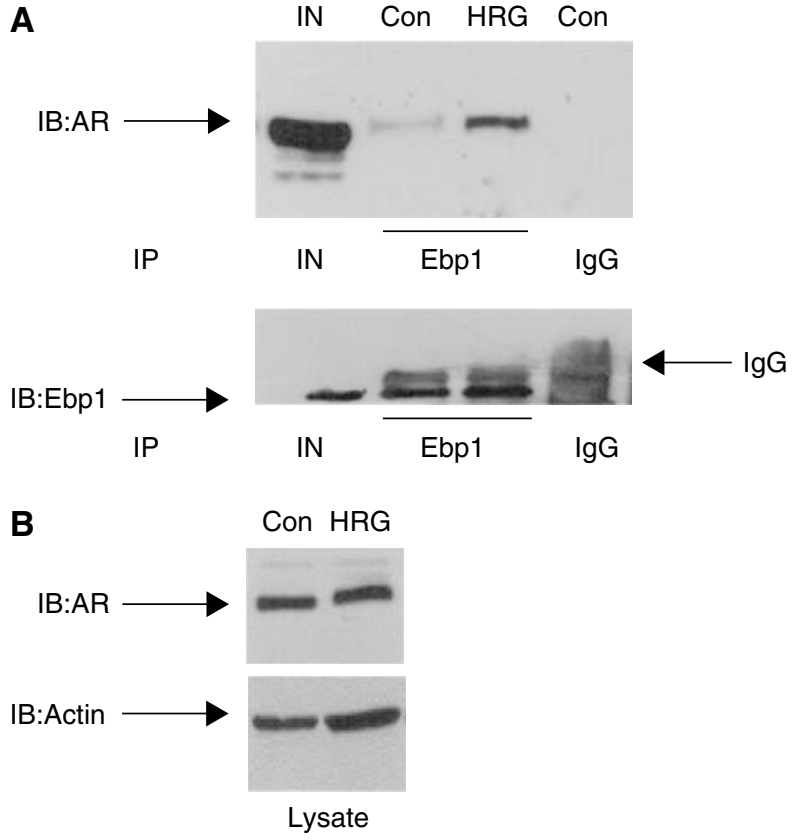

Figure 5 HRG increases the binding of AR to Ebpl in LNCaP cells. (A) $\mathrm{LNCaP}$ cells growing in complete media were switched to phenol red free RMPI 1640 containing $1 \%$ CSS and $10^{-8}$ M RI88I overnight. Cells were then incubated in the absence (Con) or presence of HRG $\beta \mid$ (HRG) $\left(20 \mathrm{ng} \mathrm{ml}^{-1}\right)$ for I h. Cell lysates were then immunoprecipitated (IP) with antibody to Ebpl (Ebpl, lanes 2 and 3) or preimmune lgG (IgG) (lane 4). Immunoblots (IB) were analysed using a monoclonal antibody to $A R$ (upper panel) or a polyclonal antibody to Ebpl (lower panel) as indicated. $\mathrm{IN}=$ input (5\% of total) used for immunoprecipitation (lane I). IgG indicates the heavy chain of IgG detected in Ebp I immunoblots of the Ebp I immunoprecipitates. (B) Effects of HRG on AR protein levels. Control (Con) or HRG treated (HRG) LNCaP cells were prepared as described in (A). Total cell lysates were resolved by SDS-PAGE, proteins transferred onto PVDF membranes, and Western blot analysis performed using monoclonal antibodies to AR or actin as indicated.

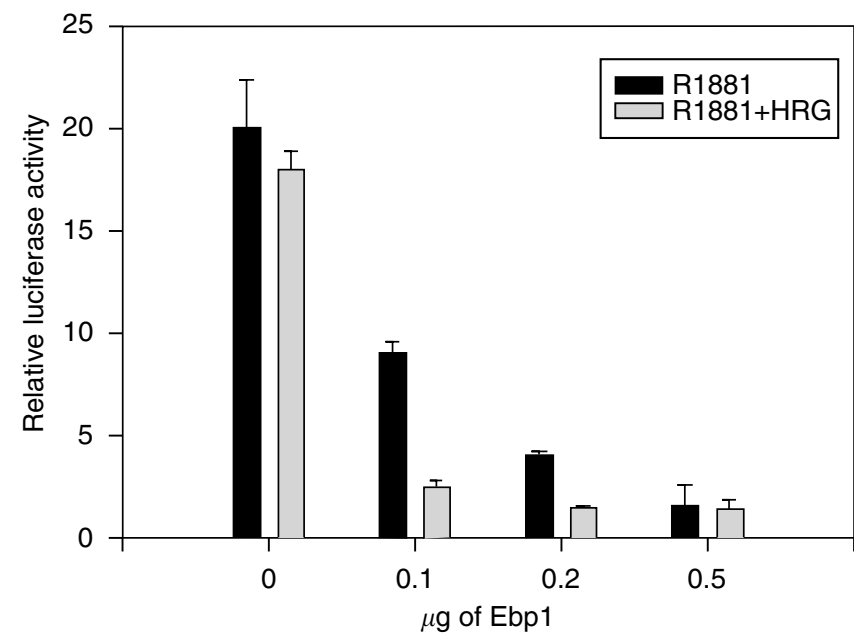

Figure 6 HRG enhances Ebp I mediated repression of the AR promoter LNCaP cells were transfected with the MMTV-luciferase reporter plasmid $(0.5 \mu \mathrm{g})$ and an ebp / expression plasmid or a pcDNA vector control at the indicated amounts. After $24 \mathrm{~h}$, cells were switched to RPMI 1640 phenolred free media with 5\% CSS and RI88I with or without HRG $\beta$ | $\left(20 \mathrm{ng} \mathrm{ml}^{-1}\right)$. Cell lysates were assayed for luciferase activity $16 \mathrm{~h}$ later. The relative luciferase activity in the absence of $R \mid 88$ I was set at I for cells that received the vector control. All activities presented in the graph were derived by comparison to that value. Neither HRG nor ebp I affected luciferase activity under these basal conditions. Each point represents mean \pm s.e. of triplicate wells. Representative of two experiments. 
fact the AR receptor is mutated in LNCaP cells, or to different transfection efficiencies of the ebp1 plasmid among the three cell lines.

Studies using siRNA demonstrated that inhibition of endogenous Ebp1 expression resulted in increased activity of an androgen regulated reporter construct in the absence of androgens. No such effect was observed in cells not expressing the AR. Thus, the increase in promoter activity in the absence of androgens was mediated via the AR. This finding suggests that Ebp1 may play a role in inhibition of AR signalling in the presence of no or extremely low levels of androgens. Ebp1 has the ability to bind DNA (Zhang and Hamburger, 2004) and histone deacetylases (Zhang et al, 2003), and we postulate that Ebp1 might reside on AR regulated promoters in the absence of androgens to inhibit transcription. ChIP assays to demonstrate Ebp1 occupancy on AR promoters are underway in the laboratory. The fact that abolition of Ebp1 protein enhanced basal, but not AR stimulated transcription, is somewhat puzzling in light of the fact that overexpression of Ebp1 inhibits R1881 stimulated, but not basal, transcription of $\mathrm{AR}$ regulated genes. It is possible that basal transcription by AR is so low in unstimulated LNCaP cells that Ebp1-mediated repression in the absence of androgens may have gone undetected. Conversely, overexpression of Ebp1 might drive Ebp1 to AR promoters in the presence of androgens. Ebp1 might then recruit HDACS, important in AR transcriptional repression (List et al, 1999), to the promoter to inhibit gene transcription. The fact that inhibition of expression of Ebp1 leads to increased transcriptional activation of AR suggests that endogenous Ebp1 may function to regulate AR signalling in prostate cancer cells.

Although Ebp1 was shown to be associated with ErbB3 in breast cancer cells (Yoo et al, 2000), the interaction of Ebp1 with ErbB3 in prostate cells had not yet been demonstrated. Here, we determined that Ebp1 could also bind ErbB3 in LNCaP cells. To the best of our knowledge, this is the first demonstration of a direct interaction between ErbB3 and the AR. It is of interest that Bonaccorsi et al (2004) recently reported the physical association and subcellular colocalisation of the EGFR with AR in PC3 cells transfected with the AR. These studies further support the concept that ErbB receptors and AR interact in vivo.

We then determined the effects of HRG on Ebp1 function. HRG treatment resulted in dissociation of Ebp1 from the ErbB3 receptor in LNCaP cells. The present studies also demonstrate that HRG regulated the interaction of Ebp1 with the AR receptor. First, we demonstrated that endogenous Ebp1 associated with endogenous $\mathrm{AR}$ in vivo. However, the efficiency of the Ebp1:AR interaction was relatively low in the absence of HRG. HRG treatment of LNCaP cells for $1 \mathrm{~h}$ enhanced the association of Ebp1 with AR in LNCaP cells. The basis of the increased association of Ebp1 with AR after HRG treatment is not known. We have found that HRG increases phosphorylation of Ebp1 in breast cancer cells (Lessor et al, 2000) and studies are underway in the laboratory to examine if enhanced phosphorylation of Ebp1 increases its binding to AR. The intracellular compartment in which $\mathrm{AR}$ and Ebp1 interact in either the presence or absence of HRG is not clear at this time. Immunofluorescence analysis in our hands indicates that both Ebp1 and AR are located in the nucleus and the cytoplasm of LNCaP cells in the absence of HRG and/or R1881 (data not shown).

The fact that HRG enhances the binding of Ebp1 to AR suggests that in the absence of HRG, Ebp1 may not optimally affect AR function. Indeed, HRG potentiated the ability of limiting amounts of Ebpl to inhibit AR promoter activity. HRG has been previously shown to inhibit growth of AR + but not AR - prostate cancer cell lines (Grasso et al, 1997b; Lyne et al, 1997). Similarly, AbreuMartin et al (1999) found that MEKK-1, a downstream mediator of HRG signalling, induced apoptosis of AR + , but not AR-, prostate cancer cell lines. We hypothesise that Ebp1 may be one mediator of the effect of $\mathrm{HRG}$ on $\mathrm{AR}$ function. In the presence of low concentrations of HRG, such as has been observed in prostate cancer tissues (Lyne et al, 1997), the activity of Ebp1 may be suboptimal, resulting in increased AR signalling. Thus, although Ebpl may be present in prostate cancer cells (Xia et al, 2001b), it may not be fully functional.

In summary, Ebp1 is a potent corepressor of AR with broad specificity. Ebp1 maintains its corepressor activity independent of cell type and promoter examined. Thus, Ebp1 joins a small but growing group of AR corepressors (Culig et al, 2003). The fact that interactions of Ebp1 and AR were regulated by HRG suggests a link between the HRG-generated growth inhibitory signals transduced through the ErbB3 receptor and the AR receptor. Further studies are needed to characterise the interactions of ErbB3, Ebp1 and AR in the progression of prostate cancer.

\section{ACKNOWLEDGEMENTS}

This work was supported in part by grants NIH R01 CA76047and R21 088882-01 (to AWH) and Intramural Funds from the Department of Pathology, University of Maryland School of Medicine. We thank Dr Olli Janne for the AR expression plasmids and the probasin reporter construct, Dr Martin Gleave for the PSA reporter construct, Dr Joseph Fondell for the MMTV-luc reporter, and Dr Angela Brodie for the PC-3 AR cell line.

\section{REFERENCES}

Abreu-Martin MT, Chari A, Palladino AA, Craft NA, Sawyers CL (1999) Mitogen-activated protein kinase kinase kinase 1 activates androgen receptor-dependent transcription and apoptosis in prostate cancer. $\mathrm{Mol}$ Cell Biol 19: 5143 - 5154

Adam RM, Kim J, Lin J, Orsola A, Zhuang L, Rice DC, Freeman MR (2002) Heparin-binding epidermal growth factor-like growth factor stimulates androgen-independent prostate tumor growth and antagonizes androgen receptor function. Endocrinology 143: $4599-4608$

Amler LC, Agus DB, LeDuc C, Sapinoso ML, Fox WD, Kern S, Lee D, Wang V, Leysens M, Higgins B, Martin J, Gerald W, Dracopoli N, CordonCardo C, Scher HI, Hampton GM (2000) Dysregulated expression of androgen-responsive and nonresponsive genes in the androgen-independent prostate cancer xenograft model CWR22-R1. Cancer Res 60: $6134-6141$

Bacus SS, Yarden Y, Oren M, Chin DM, Lyass L, Zelnick CR, Kazarov A, Toyofuku W, Gray-Bablin J, Beerli RR, Hynes NE, Nikiforov M, Haffner R, Gudkov A, Keyomarsi K (1996) Neu differentiation factor (Heregulin) activates a p53-dependent pathway in cancer cells. Oncogene 12: $2535-$ 2547
Bonaccorsi L, Carloni V, Muratori M, Formigli L, Zecchi S, Forti G, Baldi E (2004) EGF receptor (EGFR) signaling promoting invasion is disrupted in androgen-sensitive prostate cancer cells by an interaction between EGFR and androgen receptor (AR). Int J Cancer 112: 78-86

Chen CD, Welsbie DS, Tran C, Baek SH, Chen R, Vessella R, Rosenfeld MG, Sawyers CL (2004) Molecular determinants of resistance to antiandrogen therapy. Nat Med 10: 33-39

Claessens F, Verrijdt G, Schoenmakers E, Haelens A, Peeters B, Verhoeven G, Rombauts W (2001) Selective DNA binding by the androgen receptor as a mechanism for hormone-specific gene regulation. J Steroid Biochem Mol Biol 76: 23 - 30

Craft N, Shostak Y, Carey M, Sawyers CL (1999) A mechanism for hormone-independent prostate cancer through modulation of androgen receptor signaling by the HER-2/neu tyrosine kinase [see comments]. Nat Med 5: $280-285$

Culig Z, Hobisch A, Cronauer MV, Radmayr C, Trapman J, Hittmair A, Bartsch G, Klocker H (1994) Androgen receptor activation in prostatic tumor cell lines by insulin-like growth factor-I, keratinocyte growth factor, and epidermal growth factor. Cancer Res 54: 5474-5478 
Culig Z, Klocker H, Bartsch G, Steiner H, Hobisch A (2003) Androgen receptors in prostate cancer. J Urol 170: 1363-1369

De Miguel P, Royuela M, Bethencourt R, Ruiz A, Fraile B, Paniagua R (1999) Immunohistochemical comparative analysis of transforming growth factor alpha, epidermal growth factor, and epidermal growth factor receptor in normal, hyperplastic and neoplastic human prostates. Cytokine 11: $722-727$

El Sheikh SS, Domin J, Abel P, Stamp G, Lalani E (2003) Androgenindependent prostate cancer: potential role of androgen and ErbB receptor signal transduction crosstalk. Neoplasia 5: 99-109

Feldman BJ, Feldman D (2001) The development of androgen-independent prostate cancer. Nat Rev Cancer 1: 34-45

Fernandes AM, Hamburger AW, Gerwin BI (1999) Dominance of ErbB-1 heterodimers in lung epithelial cells overexpressing ErbB-2. Both ErbB-1 and ErbB-2 contribute significantly to tumorigenicity. Am J Respir Cell Mol Biol 21: $701-709$

Grasso AW, Wen D, Miller CM, Rhim JS, Pretlow TG, Kung HJ (1997a) ErbB kinases and NDF signaling in human prostate cancer cells. Oncogene 15: 2705-2716

Grasso AW, Wen D, Miller CM, Rhim JS, Pretlow TG, Kung HJ (1997b) ErbB kinases and NDF signaling in human prostate cancer cells. Oncogene 15: 2705-2716

Grossmann ME, Huang H, Tindall DJ (2001) Androgen receptor signaling in androgen-refractory prostate cancer. J Natl Cancer Inst 93: 1687- 1697

He B, Kemppainen JA, Wilson EM (2000) FXXLF and WXXLF sequences mediate the NH2-terminal interaction with the ligand binding domain of the androgen receptor. J Biol Chem 275: 22986-22994

Hernes E, Fossa SD, Berner A, Otnes B, Nesland JM (2004) Expression of the epidermal growth factor receptor family in prostate carcinoma before and during androgen-independence. Br J Cancer 90: 449-454

Holter E, Kotaja N, Makela S, Strauss L, Kietz S, Janne OA, Gustafsson JA, Palvimo JJ, Treuter E (2002) Inhibition of androgen receptor (AR) function by the reproductive orphan nuclear receptor DAX-1. Mol Endocrinol 16: 515-528

Kirschbaum MH, Yarden Y (2000) The ErbB/HER family of receptor tyrosine kinases: a potential target for chemoprevention of epithelial neoplasms. J Cell Biochem Suppl 34: 52-60

Kotaja N, Aittomaki S, Silvennoinen O, Palvimo JJ, Janne OA (2000) ARIP3 (androgen receptor-interacting protein 3) and other PIAS (protein inhibitor of activated STAT) proteins differ in their ability to modulate steroid receptor-dependent transcriptional activation. Mol Endocrinol 14: $1986-2000$

LaTulippe E, Satagopan J, Smith A, Scher H, Scardino P, Reuter V, Gerald WL (2002) Comprehensive gene expression analysis of prostate cancer reveals distinct transcriptional programs associated with metastatic disease. Cancer Res 62: 4499-4506

Lessor TJ, Yoo JY, Xia X, Woodford N, Hamburger AW (2000) Ectopic expression of the ErbB-3 binding protein ebp1 inhibits growth and induces differentiation of human breast cancer cell lines. J Cell Physiol 183: $321-329$

Leung HY, Weston J, Gullick WJ, Williams G (1997) A potential autocrine loop between heregulin-alpha and erbB-3 receptor in human prostatic adenocarcinoma. Br J Urol 79: $212-216$

List HJ, Smith CL, Rodriguez O, Danielsen M, Riegel AT (1999) Inhibition of histone deacetylation augments dihydrotestosterone induction of androgen receptor levels: an explanation for trichostatin A effects on androgen-induced chromatin remodeling and transcription of the mouse mammary tumor virus promoter. Exp Cell Res 252: 471-478

Long BJ, Grigoryev DN, Nnane IP, Liu Y, Ling YZ, Brodie AM (2000) Antiandrogenic effects of novel androgen synthesis inhibitors on hormone-dependent prostate cancer. Cancer Res 60: 6630-6640

Lyne JC, Melhem MF, Finley GG, Wen D, Liu N, Deng DH, Salup R (1997) Tissue expression of neu differentiation factor/heregulin and its receptor complex in prostate cancer and its biologic effects on prostate cancer cells in vitro. Cancer J Sci Am 3: 21-30

Mousses S, Wagner U, Chen Y, Kim JW, Bubendorf L, Bittner M, Pretlow T, Elkahloun AG, Trepel JB, Kallioniemi OP (2001) Failure of hormone therapy in prostate cancer involves systematic restoration of androgen responsive genes and activation of rapamycin sensitive signaling. Oncogene 20: 6718-6723

Olapade-Olaopa EO, Moscatello DK, MacKay EH, Horsburgh T, Sandhu DP, Terry TR, Wong AJ, Habib FK (2000) Evidence for the differential expression of a variant EGF receptor protein in human prostate cancer. Br J Cancer 82: $186-194$

Petre-Draviam CE, Cook SL, Burd CJ, Marshall TW, Wetherill YB, Knudsen KE (2003) Specificity of cyclin D1 for androgen receptor regulation. Cancer Res 63: 4903-4913

Qiu Y, Ravi L, Kung HJ (1998) Requirement of ErbB2 for signalling by interleukin-6 in prostate carcinoma cells. Nature 393: $83-85$

Sadar MD, Gleave ME (2000) Ligand-independent activation of the androgen receptor by the differentiation agent butyrate in human prostate cancer cells. Cancer Res 60: $5825-5831$

Tal-Or P, Di-Segni A, Lupowitz Z, Pinkas-Kramarski R (2003) Neuregulin promotes autophagic cell death of prostate cancer cells. Prostate 55: $147-157$

Torring N, Dagnaes-Hansen F, Sorensen BS, Nexo E, Hynes NE (2003) ErbB1 and prostate cancer: ErbB1 activity is essential for androgeninduced proliferation and protection from the apoptotic effects of LY294002. Prostate 56: $142-149$

Wang Q, Fondell JD (2001) Generation of a mammalian cell line stably expressing a tetracycline-regulated epitope-tagged human androgen receptor: implications for steroid hormone receptor research. Anal Biochem 289: 217-230

Weir HK, Thun MJ, Hankey BF, Ries LA, Howe HL, Wingo PA, Jemal A, Ward E, Anderson RN, Edwards BK (2003) Annual report to the nation on the status of cancer, 1975-2000, featuring the uses of surveillance data for cancer prevention and control. J Natl Cancer Inst 95: 1276-1299

Xia X, Cheng A, Lessor T, Zhang Y, Hamburger AW (2001a) Ebp1, an ErbB3 binding protein, interacts with $\mathrm{Rb}$ and affects $\mathrm{Rb}$ transcriptional regulation. J Cell Physiol 187: 209-217

Xia X, Lessor TJ, Zhang Y, Woodford N, Hamburger AW (2001b) Analysis of the expression pattern of Ebp1, an ErbB-3-binding protein. Biochem Biophys Res Commun 289: 240-244

Yarden Y (2001) The EGFR family and its ligands in human cancer signalling mechanisms and therapeutic opportunities. Eur J Cancer 37(Suppl 4): S3-S8

Yeh S, Lin HK, Kang HY, Thin TH, Lin MF, Chang C (1999) From HER2/ Neu signal cascade to androgen receptor and its coactivators: a novel pathway by induction of androgen target genes through MAP kinase in prostate cancer cells. Proc Natl Acad Sci USA 96: 5458-5463

Yoo JY, Wang XW, Rishi AK, Lessor T, Xia XM, Gustafson TA, Hamburger AW (2000) Interaction of the PA2G4 (EBP1) protein with ErbB-3 and regulation of this binding by heregulin. Br J Cancer 82: 683-690

Zhang Y, Hamburger AW (2004) Heregulin regulates the ability of the ErbB3-binding protein Ebp1 to bind E2F promoter elements and repress E2F-mediated transcription. J Biol Chem 279: 26126-26133

Zhang YX, Fondell JD, Wang QB, Xia XM, Cheng AW, Lu ML, Hamburger AW (2002) Repression of androgen receptor mediated transcription by the ErbB-3 binding protein, Ebp1. Oncogene 21: 5609-5618

Zhang YX, Woodford N, Xia XM, Hamburger AW (2003) Repression of E2F1-mediated transcription by the ErbB3 binding protein Ebp1 involves histone deacetylases. Nucleic Acids Res 31: 2168-2177

Ziada A, Barqawi A, Glode LM, Varella-Garcia M, Crighton F, Majeski S, Rosenblum M, Kane M, Chen L, Crawford ED (2004) The use of trastuzumab in the treatment of hormone refractory prostate cancer; phase II trial. Prostate 60: $332-337$ 